\title{
Risk Factors of Hypersensitivity to Carboplatin in Patients with Gynecologic Malignancies
}

\begin{abstract}
Yu-Hsiao Tai ${ }^{1,2}$, Yi-Jou Tai ${ }^{2,3}$, Heng-Cheng Hsu ${ }^{2,3}$, Shu-Ping Lee ${ }^{2}$, Yun-Yuan Chen ${ }^{2,4}$, Ying-Cheng Chiang ${ }^{2}$, Yu-Li Chen ${ }^{2,3}$, Chi-An Chen ${ }^{2}$ and Wen-Fang Cheng ${ }^{2,5,6 *}$

${ }^{1}$ Department of Obstetrics and Gynecology, National Cheng Kung University Hospital, Tainan, Taiwan, ${ }^{2}$ Department of Obstetrics and Gynecology, College of Medicine, National Taiwan University, Taipei, Taiwan, ${ }^{3}$ Department of Obstetrics and Gynecology, National Taiwan University Hospital Hsin-Chu Branch, Hsin-Chu, Taiwan, ${ }^{4}$ Taiwan Blood Services Foundation, Taipei, Taiwan, ${ }^{5}$ Graduate Institute of Oncology, College of Medicine, National Taiwan University, Taipei, Taiwan, ${ }^{6}$ Graduate Institute of Clinical Medicine, College of Medicine, National Taiwan University, Taipei, Taiwan
\end{abstract}

\section{OPEN ACCESS}

Edited by:

Fabrizio Marcucci,

Università degli Studi di Milano, Italy

Reviewed by:

Antonio Macciò,

Ospedale Oncologico Armando

Businco, Italy

Yuet-Kin Leung,

University of Cincinnati, United States

${ }^{*}$ Correspondence:

Wen-Fang Cheng

wenfangcheng@yahoo.com

Specialty section:

This article was submitted to Pharmacology of Anti-Cancer Drugs,

a section of the journal

Frontiers in Pharmacology

Received: 23 July 2017 Accepted: 23 October 2017

Published: 06 November 2017

Citation:

Tai Y-H, Tai Y-J, Hsu H-C, Lee S-P,

Chen $Y-Y$, Chiang $Y-C$, Chen $Y-L$,

Chen C-A and Cheng W-F (2017) Risk

Factors of Hypersensitivity to

Carboplatin in Patients with

Gynecologic Malignancies.

Front. Pharmacol. 8:800.

doi: 10.3389/fphar.2017.00800
We evaluated the prevalence of and risk factors for hypersensitivity reactions related to carboplatin, which is commonly used to treat gynecological malignancies. All women with pathologically documented ovarian, fallopian tube, or primary peritoneal cancer treated with carboplatin alone or a carboplatin-based combination chemotherapy regimen at a single hospital between January 2006 and December 2013 were retrospectively recruited. We analyzed the incidence, characteristics, risk factors, management, and outcomes of carboplatin-related hypersensitivity reactions among these patients. Among 735 eligible women, 75 (10.2\%) experienced a total of 215 carboplatin-related hypersensitivity reaction events. The annual incidence of carboplatin-related hypersensitivity reactions gradually increased from 0.88\% in 2006 to $5.42 \%$ in 2013. The incidence of carboplatin-related hypersensitivity was higher in patients with advanced stage disease $(P<0.001$, Kruskal-Wallis test), serous and mixed histological types ( $P=0.003$, Kruskal-Wallis test), malignant ascites $(P=0.009$, chi-square test), and history of other drug allergy ( $P<0.001$, chi-square test). Compared to women without hypersensitivity reactions, women who experienced hypersensitivity reactions had a significantly greater median cycle number (12 vs. $6, P<0.001$, independent sample $t$-test) and dose (6,816 vs. 3,844 mg, $P<0.001$, independent sample $t$-test). The cumulative incidence of carboplatin-related hypersensitivity reactions dramatically increased with $>8$ cycles or dose $>3,500 \mathrm{mg}$. Therefore, disease severity, histological type, malignant ascites, past drug allergies, and cumulative carboplatin dose are risk factors for carboplatin-related hypersensitivity reactions. Such reactions could potentially be reduced or prevented by slowing the infusion rate and using a desensitization protocol involving anti-allergy medications.

Keywords: ovarian cancer, chemotherapy, carboplatin, hypersensitivity, risk factor 


\section{INTRODUCTION}

Ovarian cancer is the leading cause of death from gynecological malignancies (Chiang et al., 2013), with a 5-year survival rate of $46 \%$ in the United States (92\% when localized, $73 \%$ with regional metastases, and 28\% with distant metastases) (Siegel et al., 2016). In the majority of ovarian cancer cases, primary management entails surgical staging or cytoreduction, followed by systemic chemotherapy (Ledermann et al., 2013). Platinumbased combination chemotherapy is the recommended treatment for platinum-sensitive recurrent ovarian cancer (Parmar et al., 2003; Pfisterer et al., 2006; Pujade-Lauraine et al., 2010). Parmar et al. (2003) reported that paclitaxel with platinum improves overall survival compared to conventional platinumbased chemotherapy among patients with relapsed platinumsensitive ovarian cancer. Carboplatin and pegylated liposomal doxorubicin (Pujade-Lauraine et al., 2010) or gemcitabine (Pfisterer et al., 2006) led to superior progression-free survival of patients with platinum-sensitive recurrent ovarian cancer compared to paclitaxel and carboplatin. Therefore, the preferred regimen for platinum-sensitive recurrent ovarian cancer is carboplatin combined with paclitaxel, liposomal doxorubicin, or gemcitabine.

Carboplatin is among the most commonly used chemotherapeutic agents for ovarian, fallopian tube, and primary peritoneal cancer, as it is active against these types of cancers with usually well-tolerated side effects (Ozols et al., 2003; Parmar et al., 2003; Pfisterer et al., 2006; Pujade-Lauraine et al., 2010). However, prolonged carboplatin use is associated with an increased incidence of carboplatin-related hypersensitivity reactions (Markman et al., 1999; Wang et al., 2009). Up to 16\% of patients with ovarian cancer who undergo treatment with carboplatin-containing regimens experience carboplatin-related hypersensitivity (Markman et al., 1999; Polyzos et al., 2001; Sliesoraitis and Chikhale, 2005). Though the characteristics of carboplatin-related hypersensitivity reactions are widely reported, limited data are available regarding such reactions in different types of patients.

In Taiwan, carboplatin was first introduced for the treatment of ovarian cancer in 2004. Here, we retrospectively evaluated carboplatin-related hypersensitivity reactions among patients with gynecological cancer (ovarian, fallopian tube, or primary peritoneal cancer) who received carboplatin-containing regimens at a single medical institute in Taiwan. We analyzed the relationships among the incidence, characteristics, risk factors, management, and outcomes of carboplatin-related hypersensitivity reactions in this patient population. We also provide recommendations for the continuation of treatment in ovarian cancer patients.

\section{Patients and methods}

We retrospectively recruited all women with pathologically documented ovarian, fallopian tube, or primary peritoneal cancer treated with single-agent carboplatin or a carboplatinbased combination chemotherapy regimen at National Taiwan University Hospital from January 2006 to December 2013. Patients who previously received platinum compounds were excluded. We retrieved clinical information, including age, menopausal status, cancer stage, surgical findings, chemotherapeutic treatment history, recurrence status, and survivorship, from the clinical and operative notes and discharge summaries stored in a centralized database. The research protocol was reviewed and approved by the institutional review board of the hospital. During the study period, our institution applied carboplatin (including carboplatin with cyclophosphamide or paclitaxel) as the front-line chemotherapy for women with ovarian, fallopian tube, and primary peritoneal cancer. For salvage chemotherapy in the case of recurrent disease, we administered chemotherapeutic regimens including carboplatin with paclitaxel, gemcitabine, or liposomal doxorubicin. Carboplatin-related hypersensitivity reactions were defined as symptoms and signs occurring minutes to hours after carboplatin administration. Such reactions were scored according to the Common Terminology Criteria for Adverse Events version 4.03, with severity assessed as mild (grades 1 and 2) or severe (grades 3 and 4). We also recorded the procedures applied to treat the hypersensitivity reactions and efforts to manage the side effects of carboplatin administration.

Statistical analyses were performed using the Statistical Package of Social Studies (SPSS) version 17.0 (SPSS, Inc., Chicago, IL) and SAS software version 9.4 (SAS Inc, NC, USA) for Windows. Univariate analysis for potential risk factors of carboplatin-related hypersensitivity reactions were assessed by the chi-square or Kruskal-Wallis test for categorical variables. The independent sample $t$-test was used to evaluate how carboplatin cycle and dose correlated with the incidence of hypersensitivity and severe hypersensitivity reactions. Multivariate analysis was conducted using logistic regression to estimate the association between the potential risk factors and the occurrence of hypersensitivity. All the statistical tests were two-tailed, and statistical significant level was defined as $p$-value $<0.05$.

\section{RESULTS}

A total of 735 eligible women who underwent treatment for ovarian, fallopian tube, or primary peritoneal cancer were recruited this the study; 75 (10.2\%) experienced a total of 215 carboplatin-related hypersensitivity reaction events. The yearly incidence of carboplatin-related hypersensitivity gradually increased from 0.88 in 2006 to $5.42 \%$ in 2013 (Table 1), in association with increased use of carboplatin. The total incidence of carboplatin-related hypersensitivity was $3.21 \%$ by the cycle of carboplatin.

The characteristics of the 735 patients are presented in Table 2. The median patient age was 56 years (range 16-94 years). Only 2 women are Mongolians and the other 733 women are Asian Taiwanese. A total of $412(56.1 \%)$ patients experienced spontaneous menopause, 295 (40.1\%) surgical menopause, and 28 (3.8\%) had preserved fertility. Among the 651 women (88.6\%) diagnosed with ovarian cancer, 474 (64.5\%) had stage III-IV disease. The histological type was serous in 381 patients (51.8\%) and clear cell in 148 patients (20.1\%). Optimal debulking surgery was performed in 491 patients (66.8\%).

The rate of hypersensitivity to carboplatin did not differ by patient age, menopausal status, disease entity, receipt of optimal 
TABLE 1 | The annual incidence of carboplatin-related hypersensitivity reactions in 735 women with ovarian, fallopian tube, or primary peritoneal cancers.

\begin{tabular}{lccc}
\hline Year & Hypersensitivity (No. of events) & Total cycles & Incidence (\%) \\
\hline 2006 & 6 & 674 & 0.88 \\
2007 & 13 & 860 & 1.49 \\
2008 & 23 & 833 & 2.69 \\
2009 & 27 & 868 & 3.05 \\
2010 & 47 & 914 & 4.89 \\
2011 & 42 & 938 & 4.29 \\
2012 & 10 & 801 & 1.23 \\
2013 & 47 & 820 & 5.42 \\
\hline $2006-2013$ & 215 & 6,708 & 3.21 \\
\hline
\end{tabular}

No, number.

debulking, or the amount of ascites. The rate of hypersensitivity was significantly higher among patients with advanced stage disease (III-IV) compared to patients with early stage disease (I-II) $(P<0.001$, Kruskal-Wallis test), and among patients with serous or mixed histological type compared to patients with other histological types ( $P=0.003$, Kruskal-Wallis test). We also found higher rates of hypersensitivity among patients with malignant ascites compared to patients without malignant ascites $(P=0.009$, chi-squared test), and in patients who had experienced allergic reactions to other medications or food (e.g., paclitaxel, penicillin, aspirin) compared to patients who had not experienced previous allergic reactions $(P<0.001$, chi-squared test). Furthermore, patients with carboplatin hypersensitivity had a higher incidence of previous allergic history to other cytotoxic drugs, including gemcitabine, paclitaxel, and doxorubicin, compared to those without $(12 / 75$ vs. $30 / 660, P<0.001$, chisquared test; Table 7).

We further evaluated the median carboplatin cycle and dose administered to women with and without carboplatin-related hypersensitivity. Compared to those without hypersensitivity reactions, women who experienced hypersensitivity reactions had a significantly higher median number of cycles (12 vs. 6, $P<0.001$, independent sample $t$-test) and dose (6,816 vs. $3,844 \mathrm{mg}, P<0.001$, independent sample $t$-test, Table 3$)$. The cumulative incidence of carboplatin-related hypersensitivity reactions increased with the number of carboplatin cycles (Figure 1A) and increasing dose (Figure 1B), especially at $>8$ cycles or a dose $>3,500 \mathrm{mg}$. The cumulative incidence of carboplatin-related hypersensitivity was $2 \%$ after 8 cycles, $6 \%$ after 14 cycles, $8 \%$ after 19 cycles, and $10 \%$ after 33 cycles, with a plateau beyond this cycle number (Figure 1A). The cumulative incidence of carboplatin-related hypersensitivity was $2 \%$ at $>3,500 \mathrm{mg}, 6 \%$ at $>7,500 \mathrm{mg}, 8 \%$ at $>10,000 \mathrm{mg}$, and $10 \%$ at $>16,000 \mathrm{mg}$ with a plateau beyond this dose (Figure 1B). The cumulative incidence of severe carboplatinrelated hypersensitivity was $1 \%$ after 15 cycles and $2 \%$ after 24 cycles, with a plateau beyond this cycle number, and $1 \%$ at $>7,500 \mathrm{mg}$ and $2 \%$ at $>12,500 \mathrm{mg}$, with a plateau beyond this dose (Figures 1A,B).

The symptoms and signs of the 75 women with carboplatinrelated hypersensitivity reactions are given in Table 4. All of
TABLE 2 | Characteristics of the 735 women receiving carboplatin-based chemotherapy.

\begin{tabular}{|c|c|c|c|c|c|}
\hline \multirow[t]{2}{*}{ Characteristics } & \multicolumn{2}{|c|}{ Hypersensitivity (n) } & \multirow[t]{2}{*}{ Total $(n)$} & \multirow[t]{2}{*}{ Prevalence (\%) } & \multirow[t]{2}{*}{$p$-value } \\
\hline & Positive & Negative & & & \\
\hline \multicolumn{6}{|l|}{ AGE (y/o) } \\
\hline$<45$ & 14 & 117 & 131 & 10.7 & 0.928 \\
\hline $45-65$ & 45 & 411 & 456 & 9.9 & \\
\hline$>65$ & 16 & 132 & 148 & 10.8 & \\
\hline \multicolumn{6}{|l|}{ MENOPAUSE } \\
\hline Yes Surgical & 34 & 261 & 295 & 11.5 & 0.152 \\
\hline Spontaneous & 41 & 371 & 412 & 10.0 & \\
\hline No & 0 & 28 & 28 & 0 & \\
\hline \multicolumn{6}{|l|}{ DISEASE ENTITY } \\
\hline Ovarian & 63 & 588 & 651 & 9.7 & 0.416 \\
\hline $\begin{array}{l}\text { Primary } \\
\text { peritoneal }\end{array}$ & 11 & 65 & 76 & 14.5 & \\
\hline Tubal & 1 & 7 & 8 & 12.5 & \\
\hline \multicolumn{6}{|l|}{ STAGE } \\
\hline I & 7 & 207 & 214 & 3.3 & $<0.001$ \\
\hline ॥ & 2 & 45 & 47 & 4.3 & \\
\hline III & 53 & 333 & 386 & 13.7 & \\
\hline IV & 13 & 75 & 88 & 14.8 & \\
\hline \multicolumn{6}{|l|}{ HISTOLOGY } \\
\hline Serous & 57 & 324 & 381 & 15.0 & 0.003 \\
\hline Clear cell & 6 & 142 & 148 & 4.1 & \\
\hline Endometrioid & 6 & 88 & 94 & 6.4 & \\
\hline Mucinous & 1 & 36 & 37 & 2.7 & \\
\hline Transitional cell & 0 & 6 & 6 & 0 & \\
\hline Carcinosarcomas & 1 & 16 & 17 & 5.9 & \\
\hline Mixed type & 4 & 35 & 39 & 10.3 & \\
\hline Others & 0 & 13 & 13 & 0 & \\
\hline \multicolumn{6}{|c|}{ OPTIMAL SURGERY } \\
\hline Yes & 43 & 448 & 491 & 8.8 & 0.066 \\
\hline No & 32 & 212 & 244 & 13.1 & \\
\hline \multicolumn{6}{|l|}{ ASCITES (ml) } \\
\hline Mild $(<500)$ & 40 & 409 & 449 & 8.9 & 0.232 \\
\hline $\begin{array}{l}\text { Moderate } \\
(500-1,000)\end{array}$ & 8 & 43 & 51 & 15.7 & \\
\hline Large $(>1,000)$ & 27 & 208 & 235 & 11.5 & \\
\hline \multicolumn{6}{|c|}{ ASCITIC MALIGNANT CELLS } \\
\hline Yes & 52 & 353 & 405 & 12.8 & 0.009 \\
\hline No & 23 & 307 & 330 & 7.0 & \\
\hline \multicolumn{6}{|c|}{ DRUG OR FOOD ALLERGIC HISTORY } \\
\hline Yes & 27 & 117 & 144 & 18.8 & $<0.001$ \\
\hline No & 48 & 543 & 591 & 8.1 & \\
\hline
\end{tabular}

these reported symptoms and signs occurred within an hour of initiating carboplatin administration. Among these 75 women, $16(21.3 \%)$ experienced severe hypersensitivity reactions (grades 3 and 4). The most commonly exhibited symptom/sign was hypotension (10.7\%), followed by anaphylaxis $(8.0 \%)$ and desaturation (6.7\%). A total of 59 women experienced mild signs and symptoms (grades 1 and 2), including skin rash (69.3\%), skin itching (64.0\%), facial flushing (56.0\%), dyspnea 
(41.3\%), chest pain $(25.3 \%)$, tachycardia (18.7\%), paresthesias (16.0\%), abdominal cramping (13.3\%), and diaphoresis (12.0\%). Upon the occurrence of symptoms and signs, carboplatin infusion was immediately stopped. These patients were provided with intravenous fluid infusion and medications, including corticosteroids, antihistamines, and oxygen application. In 74 of the 75 patients, the hypersensitivity reactions subsided within several minutes to several hours after onset, and the patients recovered without any sequelae. Sixty-one of these patients were re-challenged with carboplatin following their initial hypersensitivity reaction. However, 48 (78.7\%) of these patients developed repeated hypersensitivity reactions, even after pre-treatment with intravenous antihistamines, $\mathrm{H} 2$ blocker, and corticosteroids prior to carboplatin re-administration.

One patient who experienced a carboplatin-related hypersensitivity reaction progressed to respiratory arrest and died. This patient developed hypersensitivity reactions during her 23rd cycle of carboplatin administration at a cumulative dose of approximately $14,000 \mathrm{mg}$. She experienced skin rash, tachycardia, and dyspnea $13 \mathrm{~min}$ after starting the infusion. Tachycardia and dyspnea persisted despite treatment with hydration, antihistamines, corticosteroids, and an oxygen mask. Cardiopulmonary resuscitation and endotracheal intubation were performed, but the patient finally expired due to respiratory failure.

TABLE 3 | Median cycle and dose of carboplatin administered to women with or without hypersensitivity reactions.

\begin{tabular}{lccc}
\hline & \multicolumn{2}{c}{ Hypersensitivity reactions } & \multirow{2}{*}{$\boldsymbol{P}^{*}$} \\
\cline { 2 - 3 } & Yes $(\boldsymbol{n}=\mathbf{7 5})$ & No $(\boldsymbol{n}=\mathbf{6 6 0})$ & \\
\hline Median cycle (range) & $12(1-42)$ & $6(1-63)$ & $<0.001$ \\
Median dose (mg) (range) & $6,816(328-23,707)$ & $3,844(150-30,014)$ & $<0.001$ \\
\hline
\end{tabular}

N, patient number; *By independent sample t-test.
Evaluating whether any factors can predict carboplatin hypersensitivity, we identified a high correlation with the carboplatin cycle and cumulative dose (Pearson correlation coefficient 0.9318). Thus, we chose the cumulative carboplatin dose for the analysis to avoid interference between the highly correlated factors. As shown in Table 6, drug or food history (odd ratios [OR] 2.340, 95\% confidence interval [CI] 1.374-3.984, $P=0.0018$ ), malignant ascites (OR 1.895, 95\% CI 1.108-3.241, $P=0.0196)$, and cumulative carboplatin dose $(4,000-4,999$ mg: OR 5.507, 95\% CI 1.796-16.887, $P=0.0028 ; 5,000-9,999$ mg: OR 7.244, 95\% CI 2.740-19.149, $P<0.0001 ; \geq 10,000$ mg: OR 8.461, 95\% CI 3.007-23.806, $P<0.0001 ; P$ for trend $<0.0001)$ significantly positively correlated with carboplatin hypersensitivity.

\section{DISCUSSION}

Our results revealed carboplatin-related hypersensitivity reactions in 1 out of 10 women treated with a carboplatincontaining regimen for ovarian, fallopian tube, or primary peritoneal cancer. This was the largest series of gynecological malignancies investigated for carboplatin-related hypersensitivity reactions. We found a higher incidence of carboplatin-related hypersensitivity among patients with advanced disease (stage III-IV) with serous carcinoma and malignant ascites. These differences were likely related to the fact that patients with advanced disease, serous carcinoma, and ascetic malignant cells required significantly more carboplatin treatment cycles and higher carboplatin doses.

Our findings also suggest that a history of drug or food allergies is a predictive factor for carboplatin-related hypersensitivity. Prior investigations reported that a history of drug allergies correlates with the incidence of carboplatinrelated hypersensitivity reactions (Libra et al., 2003; Sliesoraitis and Chikhale, 2005). In our study, 36\% of the patients with carboplatin-related hypersensitivity reactions had a history of
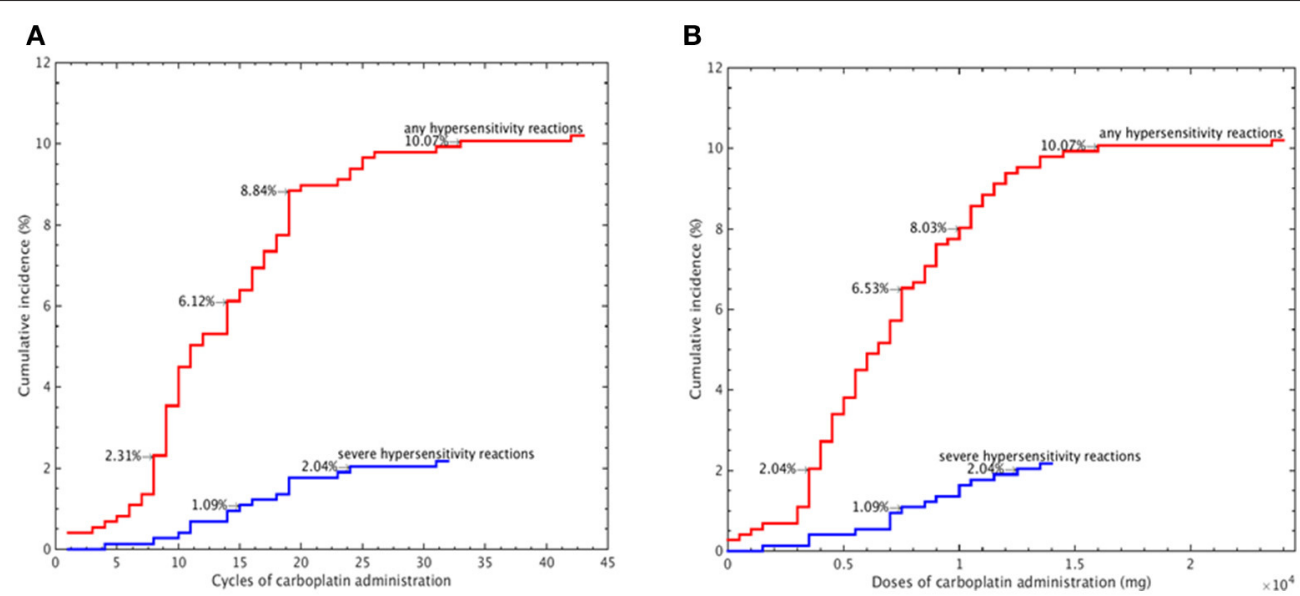

FIGURE 1 | Cumulative incidence of carboplatin-related hypersensitivity reactions according to the carboplatin administration cycle number (A) and carboplatin dose (B). 
TABLE 4 | Symptoms and signs of 75 patients with hypersensitivity reactions to carboplatin.

\begin{tabular}{lc}
\hline Symptoms and signs & Number of Events* (\%) \\
\hline MILD REACTIONS & 59 \\
Patient number & $52(69.3)$ \\
Skin rash & $48(64.0)$ \\
Skin itching & $42(56.0)$ \\
Facial flushing & $31(41.3)$ \\
Dyspnea & $19(25.3)$ \\
Chest pain & $14(18.7)$ \\
Tachycardia & $12(16.0)$ \\
Paresthesias & $10(13.3)$ \\
Abdominal cramping & $9(12.0)$ \\
Diaphoresis & $8(10.7)$ \\
Nausea/vomiting & $7(9.3)$ \\
Dizziness & $5(6.7)$ \\
Diarrhea & $5(6.7)$ \\
Cough & $4(5.3)$ \\
Chills & $2(2.7)$ \\
Wheezing & $2(2.7)$ \\
Fatigue & $1(1.3)$ \\
Headache & $16(10.7)$ \\
SEVERE REACTIONS & $1(1.3)$ \\
Hyatient number & $5(6.7)$ \\
Anaphylaxis & $1(1.3)$ \\
Desaturation & \\
Seizure & $1.3)$ \\
\hline Respiratory arrest & \\
\hline
\end{tabular}

*Some patients had more than one symptoms and signs.

drug allergies. Patients with prior drug or food allergies should be closely monitored during carboplatin administration.

Table 5 reports the incidences of carboplatin hypersensitivity and patient characteristics reported in various investigations, including the present study. The incidence of carboplatin hypersensitivity ranges from 8 to $11 \%$ (Libra et al., 2003; ConfinoCohen et al., 2005; Schwartz et al., 2007; Koshiba et al., 2009). The incidence of severe carboplatin-related hypersensitivity reactions ranges from 2 to $6 \%$, with the lowest incidence occurring in our study. Among cases developing carboplatinrelated hypersensitivity reactions, the median number of cycles is $9-12$, and the median doses $6,000-8,000 \mathrm{mg}$. Reported risk factors for carboplatin-related hypersensitivity reactions include carboplatin cycle/dose, platinum-free interval, and history of drug allergies (Libra et al., 2003; Confino-Cohen et al., 2005; Schwartz et al., 2007; Koshiba et al., 2009). Our present findings confirmed these factors and identified other risk factors, including advanced disease, serous histology, and the presence of malignant ascites. These risk factors can help identify patients at greater risk of developing hypersensitivity.

The incidence of carboplatin-related hypersensitivity correlated with cycle number and dose, with the first episode occurring at a median of 12 cycles and $6,816 \mathrm{mg}$. Similarly, a
Japanese study found that carboplatin hypersensitivity occurred at a median of 11.5 cycles and 8,084.5 mg (Koshiba et al., 2009). Markman et al. (Markman, 2007) reported that carboplatinrelated hypersensitivity reactions rarely occur before cycle 6 or $<3,000 \mathrm{mg}$ of drug delivery, with the first episode most commonly occurring with carboplatin administration in the second-line setting, and carboplatin administration beyond 9 cycles or $6,000 \mathrm{mg}$ increasing the risk of severe hypersensitivity reactions, similar to our present observations. Previous data indicated that prior carboplatin exposure is the primary risk factor for carboplatin-related hypersensitivity reactions among all patients (Navo et al., 2006; Koshiba et al., 2009). Similarly, Kandel et al. (2005) reported that all carboplatinassociated hypersensitivity reactions occur in patients with prior carboplatin exposure, and Tamiya et al. (2011) found that the number of treatment cycles with platinum-containing antineoplastic agents significantly correlates with the incidence of related hypersensitivity reactions. Overall, the evidence supports that the number of carboplatin cycles and dose are the principal risk factors for carboplatin-related hypersensitivity reactions, and that caution is warranted in cases of carboplatin infusion beyond 8 cycles or 3,500 mg.

Severe carboplatin-related hypersensitivity reactions are uncommon. Koshiba et al. (2009) reported a 3.54\% rate of severe carboplatin-related hypersensitivity reactions, which is similar to our rate of $2.2 \%$. However, severe carboplatin-related hypersensitivity symptoms can be fatal, as in one of our cases. Thus, the risk of severe carboplatin-related hypersensitivity may influence its usage in gynecological cancers, and it is important to develop protocols to reduce this risk, especially the risk of severe reactions.

Carboplatin-related hypersensitivity reactions sometimes result in premature discontinuation of treatment (Schwartz et al., 2007). One-fifth of these patients never attempt carboplatin reinfusion, even after only mild hypersensitivity reactions (Gadducci et al., 2008). Shah et al. (Bruchim et al., 2014) demonstrated safe re-treatment with carboplatin using a desensitization protocol to reduce repeated hypersensitivity reactions, and Koshiba et al. (2009) reported the successful re-treatment of the majority of patients with previous carboplatin-related hypersensitivity reactions. Altwerger et al. also successfully developed a platinum desensitization protocol for patients with either a positive carboplatin skin test or a history of prior carboplatin hypersensitivity (Altwerger et al., 2017). In the present study, 61 patients with carboplatin-related hypersensitivity were rechallenged with carboplatin. With a lower carboplatin infusion rate and pre-treatment with anti-allergy drugs, all of these patients were able to tolerate re-treatment with carboplatin, although $48(78.7 \%)$ of these 61 patients developed hypersensitivity again despite the desensitization protocol. Several methods have been developed to prevent carboplatin-related hypersensitivity reactions (Libra et al., 2003; Confino-Cohen et al., 2005; Gomez et al., 2009; Greene et al., 2010; Takase et al., 2015; Shah et al., 2016). Genc et al. (2012) demonstrated that carboplatin treatment can be continued successfully in patients whose hypersensitivity reactions are managed in a timely fashion. Abe et al. (2010) described the 
TABLE 5 | The comparison between other countries and ours about characteristics of the patients receiving carboplatin-based chemotherapy and risk factors of carboplatin-related hypersensitivity reactions.

\begin{tabular}{|c|c|c|c|c|c|}
\hline Country & Taiwan & USA (18) & Japan (19) & Italy (20) & Israel (21) \\
\hline Number of patients & 735 & 585 & 113 & 112 & 254 \\
\hline $\begin{array}{l}\text { Incidence of hypersensitivity } \\
\text { reactions }\end{array}$ & $\begin{array}{l}10.2 \% \\
(75 / 735)\end{array}$ & $\begin{array}{l}11.6 \% \\
(68 / 585)\end{array}$ & $\begin{array}{l}8.85 \% \\
(10 / 113)\end{array}$ & $\begin{array}{l}8.0 \% \\
(9 / 112)\end{array}$ & $\begin{array}{l}9.1 \% \\
(23 / 254)\end{array}$ \\
\hline $\begin{array}{l}\text { Incidence of severe } \\
\text { hypersensitivity reactions }\end{array}$ & $\begin{array}{l}2.18 \% \\
(16 / 735)\end{array}$ & $\begin{array}{l}6.67 \% \\
(39 / 585)\end{array}$ & $\begin{array}{l}3.54 \% \\
(4 / 113)\end{array}$ & $\begin{array}{l}2.6 \% \\
(3 / 112)\end{array}$ & $\begin{array}{l}5.91 \% \\
(15 / 254)\end{array}$ \\
\hline Death & 1 & No & No & No & No \\
\hline $\begin{array}{l}\text { Carboplatin hypersensitivity in } \\
\text { carboplatin retreatment }\end{array}$ & $61 / 75$ & $10 / 68$ & $9 / 10$ & $\mathrm{n} / \mathrm{a}$ & $20 / 23$ \\
\hline $\begin{array}{l}\text { Median cycle for the first } \\
\text { carboplatin-related } \\
\text { hypersensitivity reactions }\end{array}$ & 12 & $\mathrm{n} / \mathrm{a}$ & 11.5 & $\mathrm{n} / \mathrm{a}$ & 9 \\
\hline $\begin{array}{l}\text { Median dose for the first } \\
\text { carboplatin-related } \\
\text { hypersensitivity reactions (mg) }\end{array}$ & 6,816 & $\mathrm{n} / \mathrm{a}$ & $8084.5 \mathrm{mg}$ & $\mathrm{n} / \mathrm{a}$ & $\mathrm{n} / \mathrm{a}$ \\
\hline Risk factor & $\begin{array}{l}\text { 1. Carboplatin cycle or } \\
\text { dose } \\
\text { 2. Advanced stages (stages } \\
\text { III and IV) } \\
\text { 3. Serous histologic type } \\
\text { 4. malignant ascites } \\
\text { 5. Drug or food allergic } \\
\text { history }\end{array}$ & $\begin{array}{l}\text { 1. Carboplatin cycle } \\
\text { 2. Platinum-free interval of } \\
\text { over } 12 \text { months } \\
\text { 3. Drug allergies }\end{array}$ & $\begin{array}{l}\text { 1. Carboplatin cycle, dose } \\
\text { or number of regimens } \\
\text { 2. Diagnosis of ovarian } \\
\text { cancer }\end{array}$ & $\mathrm{n} / \mathrm{a}$ & Carboplatin cycle \\
\hline
\end{tabular}

n/a, not available.

TABLE 6 | Multivariate analyses of clinical parameters for carboplatin-related hypersensitivity reactions.

\begin{tabular}{|c|c|c|}
\hline Variable & Odds ratio (95\% confidence interval) & $P$-value* \\
\hline \multicolumn{3}{|l|}{ AGE, YEARS } \\
\hline$<65$ & 1 & \\
\hline$>65$ & $0.994(0.538-1.834)$ & 0.983 \\
\hline \multicolumn{3}{|c|}{ DRUG OR FOOD ALLERGY HISTORY } \\
\hline No & 1 & \\
\hline Yes & $2.340(1.374-3.984)$ & 0.0018 \\
\hline \multicolumn{3}{|c|}{ MALIGNANT ASCITES } \\
\hline No & 1 & \\
\hline Yes & $1.895(1.108-3.241)$ & 0.0196 \\
\hline \multicolumn{3}{|c|}{ CARBOPLATIN DOSE, mg ${ }^{\#}$} \\
\hline$<3,000$ & 1 & \\
\hline $3,000-3,999$ & $2.462(0.817-7.416)$ & 0.109 \\
\hline $4,000-4,999$ & $5.507(1.796-16.887)$ & 0.0028 \\
\hline $5,000-9,999$ & $7.244(2.740-19.149)$ & $<0.0001$ \\
\hline$>10,000$ & $8.461(3.007-23.806)$ & $<0.0001$ \\
\hline
\end{tabular}

${ }^{*}$ Multivariate analysis, ${ }^{\#} P$ for trend $<0.0001$.

use of cisplatin as an alternative to carboplatin. However, other studies have reported severe hypersensitivity reactions to cisplatin in patients with prior carboplatin hypersensitivity reactions (Zweizig et al., 1994; Dizon et al., 2002).

Carboplatin induces DNA double strand breaks, which are repaired by BRCA recombination repair enzymes. BRCA1/2 mutation has been reported to correlate with carboplatin hypersensitivity (Altwerger et al., 2017). Furthermore,
TABLE 7 | The drug or food allergy in 735 patients receiving carboplatin-based chemotherapy.

\begin{tabular}{|c|c|c|c|}
\hline \multirow[t]{2}{*}{ Allergy } & \multicolumn{2}{|c|}{ Carboplatin hypersensitivity } & \multirow[t]{2}{*}{$P$-value $\#$} \\
\hline & Yes $(n=75)$ & No $(n=660)$ & \\
\hline \multicolumn{4}{|c|}{ CONTRAST MEDIUM } \\
\hline Yes & 5 & 22 & \\
\hline No & 70 & 638 & 0.146 \\
\hline \multicolumn{4}{|c|}{ OTHER CYTOTOXIC DRUGS } \\
\hline Yes & 12 & 30 & \\
\hline No & 63 & 630 & $<0.001$ \\
\hline \multicolumn{4}{|c|}{ ANTIBIOTICS } \\
\hline Yes & 5 & 39 & \\
\hline No & 70 & 621 & 0.790 \\
\hline \multicolumn{4}{|c|}{ OTHER DRUGS } \\
\hline Yes & 13 & 66 & \\
\hline No & 62 & 594 & 0.052 \\
\hline \multicolumn{4}{|l|}{ FOOD } \\
\hline Yes & 0 & 5 & \\
\hline No & 75 & 655 & 0.450 \\
\hline Total ${ }^{\star}$ & 35 & 162 & \\
\hline
\end{tabular}

*One woman may have had an allergy to at least one kind of drug or food, "chi-squared test.

Moon et al. reported that the majority (93\%) of patients with a BRCA1/2 mutation develop carboplatin hypersensitivity, and that BRCA1/2 mutation is an independent risk factor for the development of carboplatin hypersensitivity. (Moon et al., 
2013) BRCA1/2 mutation patients had early-onset carboplatin hypersensitivity at a lower cumulative exposure. The BRCA status of our patients was not available for further analysis in this retrospective study. However, basophil activation has been observed in patients with a history of severe carboplatin hypersensitivity reaction (Iwamoto et al., 2014). Whether, BRCA $1 / 2$ and HRD-related genes modulate Th2 gene expression and increase specific IgE to carboplatin is still unknown. Recently, more and more patients with ovarian cancer have been receiving genetic counseling and testing, including BRCA1/2 and the other heterologous recombinant deficient (HRD) genes, because of the development of PAPP inhibitors. It would be worthwhile to evaluate the correlation between other HRD genes and carboplatin hypersensitivity.

A history of drug or food allergy, the presence of malignant ascites, and the cumulative carboplatin dose are three independent predictive factors of carboplatin hypersensitivity in this survey. A cumulative carboplatin dose $>4,000 \mathrm{mg}$ significantly positively correlated with carboplatin hypersensitivity. Close monitoring is warranted for patients with any one of these three risk factors who are receiving carboplatin. Reducing the carboplatin infusion rate and employing desensitization protocols with anti-allergy medications are especially important for patients at high risk of carboplatin hypersensitivity to detect the hypersensitivity early and avoid severe hypersensitivity without compromising the efficacy of the antineoplastic regimen. It would be worthwhile to design a perspective trial that could randomize patients with carboplatin hypersensitivity to receive an alternative regimen without carboplatin. The results of the trial could

\section{REFERENCES}

Abe, A., Ikawa, H., and Ikawa, S. (2010). Desensitization treatment with cisplatin after carboplatin hypersensitivity reaction in gynecologic cancer. J. Med. Invest. 57, 163-167. doi: 10.2152/jmi.57.163

Altwerger, G., Gressel, G. M., English, D. P., Nelson, W. K., Carusillo, N., Silasi, D. A., et al. (2017). Platinum desensitization in patients with carboplatin hypersensitivity: a single-institution retrospective study. Gynecol. Oncol. 144, 77-82. doi: 10.1016/j.ygyno.2016.09.027

Bruchim, I., Goldberg, A., Fishman, A., and Confino-Cohen, R. (2014). Carboplatin hypersensitivity: evaluation and successful desensitization protocol. Immunotherapy 6, 905-912. doi: 10.2217/imt.14.71

Chiang, Y. C., Chen, C. A., Chiang, C. J., Hsu, T. H., Lin, M. C., You, S. L., et al. (2013). Trends in incidence and survival outcome of epithelial ovarian cancer: 30-year national population-based registry in Taiwan. J. Gynecol. Oncol. 24, 342-351. doi: 10.3802/jgo.2013.24.4.342

Confino-Cohen, R., Fishman, A., Altaras, M., and Goldberg, A. (2005). Successful carboplatin desensitization in patients with proven carboplatin allergy. Cancer 104, 640-643. doi: 10.1002/cncr.21168

Dizon, D. S., Sabbatini, P. J., Aghajanian, C., Hensley, M. L., and Spriggs, D. R. (2002). Analysis of patients with epithelial ovarian cancer or fallopian tube carcinoma retreated with cisplatin after the development of a carboplatin allergy. Gynecol. Oncol. 84, 378-382. doi: 10.1006/gyno.2001.6519

Gadducci, A., Tana, R., Teti, G., Zanca, G., Fanucchi, A., and Genazzani, A. R. (2008). Analysis of the pattern of hypersensitivity reactions in patients receiving carboplatin retreatment for recurrent ovarian cancer. Int. J. Gynecol. Cancer. 18, 615-620. doi: 10.1111/j.1525-1438.2007.01063.x

Genc, D. B., Canpolat, C., and Berrak, S. G. (2012). Clinical features and management of carboplatin-related hypersensitivity reactions in answer whether carboplatin hypersensitivity patients have similar chemo-responses in non-platinum regimens without compromising the efficacy of the antineoplastic regimens of these patients.

In conclusion, in our present population of women with gynecological malignancies treated with platinumbased chemotherapy, 1 in 10 developed carboplatin-related hypersensitivity reactions. Risk factors for these reactions included advanced disease, serous histological type, malignant ascites, and history of drug allergies. Close monitoring is warranted for patients with a history of drug or food allergy, malignant ascites, or cumulative carboplatin dose $>4,000 \mathrm{mg}$. Strategies for preventing carboplatin-related hypersensitivity include reducing the carboplatin infusion rate and employing desensitization protocols with anti-allergy medications. In addition, the development of a new generation of platinum cytotoxic drugs to avoid hypersensitivity reactions is warranted.

\section{ETHICS STATEMENT}

This study was approved by the Research Ethics Committee at the National Taiwan University Hospital (201706023RINC).

\section{AUTHOR CONTRIBUTIONS}

Study conception and design: Y-HT and W-FC. Acquisition of data: Y-HT, S-PL, Y-CC, Y-LC. Analysis and interpretation of data: Y-HT, Y-JT, H-CH, Y-YC, and W-FC. Drafting of manuscript: Y-HT and W-FC. Critical revision: C-AC.

pediatric low-grade glioma. Supportive. Care. Cancer 20, 385-393. doi: 10.1007/s00520-011-1123-y

Gomez, R., Harter, P., Lück, H. J., Traut, A., Kommoss, S., Kandel, M., et al. (2009). Carboplatin hypersensitivity: does introduction of skin test and desensitization reliably predict and avoid the problem? A prospective single-center study. Int. J. Gynecol. Cancer 19, 1284-1287. doi: 10.1111/IGC.0b013e3181a418ff

Greene, D. P., Ferriss, J. S., and Jazaeri, A. A. (2010). Cisplatin administration following carboplatin desensitization failure in primary peritoneal cancer: a brief report. Cancer. Chemother. Pharmacol. 66, 265-267. doi: 10.1007/s00280-009-1159-6

Iwamoto, T., Hirai, H., Yamaguchi, N., Kobayashi, N., Sugimoto, H., and Tabata, T. (2014). Carboplatin-induced severe hypersensitivity reaction: role of IgE-dependent basophil activation and FceRI. Cancer Sci. 105, 1472-1479. doi: $10.1111 /$ cas. 12538

Kandel, M. J., Loehr, A., Harter, P., Traut, A., Gnauert, K., and du Bois, A. (2005) Cisplatinum rechallenge in relapsed ovarian cancer patients with platinum reinduction therapy and carboplatin hypersensitivity. Int. J. Gynecol. Cancer. 15, 780-784. doi: 10.1111/j.1525-1438.2005.00136.x

Koshiba, H., Hosokawa, K., Kubo, A., Miyagi, Y., Oda, T., Miyagi, Y., et al. (2009). Incidence of carboplatin-related hypersensitivity reactions in Japanese patients with gynecologic malignancies. Int. J. Gynecol. Cancer. 19, 460-465. doi: 10.1111/IGC.0b013e3181a1bf2e

Ledermann, J. A., Raja, F. A., Fotopoulou, C., Gonzalez-Martin, A., Colombo, N., Sessa, C., et al. (2013). Newly diagnosed and relapsed epithelial ovarian carcinoma: ESMO Clinical Practice Guidelines for diagnosis, treatment and follow-up. Ann. Oncol. 24, 24-32. doi: 10.1093/annonc/mdt333

Libra, M., Sorio, R., Buonadonna, A., Berretta, M., Stefanovski, P., Toffoli, G., et al. (2003). Cisplatin may be a valid alternative approach in ovarian carcinoma with carboplatin hypersensitivity. Report of three cases. Tumori 89, 311-313. 
Markman, M. (2007). The dilemma of carboplatin-associated hypersensitivity reactions in ovarian cancer management. Gynecol. Oncol. 107, 163-165. doi: 10.1016/j.ygyno.2007.06.016

Markman, M., Kennedy, A., Webster, K., Elson, P., Peterson, G., Kulp, B., et al. (1999). Clinical features of hypersensitivity reactions to carboplatin. J. Clin. Oncol. 17, 1141-1145. doi: 10.1200/JCO.1999.17.4.1141

Moon, D. H., Lee, J. M., Noonan, A. M., Annunziata, C. M., Minasian, L., Houston, N., et al. (2013). Deleterious BRCA1/2 mutation is an independent risk factor for carboplatin hypersensitivity reactions. Br. J. Cancer 109, 1072-1078. doi: 10.1038/bjc.2013.389

Navo, M., Kunthur, A., Badell, M. L., Coffer, L. W. II., Markman, M., Brown, J., et al. (2006). Evaluation of the incidence of carboplatin hypersensitivity in cancer patients. Gynecol. Oncol. 103, 608-613. doi: 10.1016/j.ygyno.2006.04.002

Ozols, R. F., Bundy, B. N., Greer, B. E., Fowler, J. M., Clarke-Pearson, D., Burger, R. A., et al. (2003). Phase III trial of carboplatin and paclitaxel compared with cisplatin and paclitaxel on patients with optimally resected stage III ovarian cancer: a gynecologic oncology group study. J. Clin. Oncol. 21, 3194-3200. doi: 10.1200/JCO.2003.02.153

Parmar, M. K., Ledermann, J. A., Colombo, N., du Bois, A., Delaloye, J. F., Kristensen, G. B., et al. (2003). Paclitaxel plus platinum-based chemotherapy vs. conventional platinum-based chemotherapy in women with relapsed ovarian cancer: the ICON4/AGO-OVAR-2.2 trial. Lancet 361, 2099-2106. doi: 10.1016/S0140-6736(03)13718-X

Pfisterer, J., Plante, M., Vergote, I., du Bois, A., Hirte, H., Lacave, A. J., et al. (2006). Gemcitabine plus carboplatin compared with carboplatin in patients with platinum-sensitive recurrent ovarian cancer: An intergroup trial of the AGOOVAR, the NCIC CTG, and the EORTC GCG. J. Clin. Oncol. 24, 4699-4707. doi: 10.1200/JCO.2006.06.0913

Polyzos, A., Tsavaris, N., Kosmas, C., Arnaouti, T., Kalahanis, N., Tsigris, C., et al. (2001). Hypersensitivity reactions to carboplatin administration are common but not always severe: a 10-year experience. Oncology 61, 129-133. doi: $10.1159 / 000055363$

Pujade-Lauraine, E., Wagner, U., Aavall-Lundqvist, E., Gebski, V., Heywood, M., Vasey, P. A., et al. (2010). Pegylated liposomal doxorubicin and carboplatin compared with paclitaxel and carboplatin for patients with platinumsensitive ovarian cancer in late relapse. J. Clin. Oncol. 28, 3323-3329. doi: 10.1200/JCO.2009.25.7519
Schwartz, J. R., Bandera, C., Bradley, A., Brard, L., Legare, R., Granai, C. O., et al. (2007). Does the platinum-free interval predict the incidence or severity of hypersensitivity reactions to carboplatin? The experience from Women and Infants' Hospital. Gynecol. Oncol. 105, 81-83. doi: 10.1016/j.ygyno.2006.10.047

Shah, A. C., Minturn, J. E., Li, Y., Belasco, J. B., Phillips, P. C., Kang, T. I., et al. (2016). Carboplatin rechallenge after hypersensitivity reactions in pediatric patients with low-grade glioma. Pediatric. Blood. Cancer 63, 21-26. doi: $10.1002 /$ pbc. 25697

Siegel, R. L., Miller, K. D., and Jemal, A. (2016). Cancer statistics, 2016. CA. Cancer. J. Clin. 66, 7-30. doi: 10.3322/caac.21332

Sliesoraitis, S., and Chikhale, P. J. (2005). Carboplatin hypersensitivity. Int. J. Gynecol. Cancer 15, 13-18. doi: 10.1111/j.1048-891x.2005.14401.x

Takase, N., Matsumoto, K., Onoe, T., Kitao, A., Tanioka, M., Kikukawa, Y., et al. (2015). 4-step 4-h carboplatin desensitization protocol for patients with gynecological malignancies showing platinum hypersensitivity: a retrospective study. Int. J. Clin. Oncol. 20, 566-573. doi: 10.1007/s10147-014-0731-1

Tamiya, M., Kuhara, H., Hirashima, T., Kondo, Y., Santo, M., Morishita, N., et al. (2011). Hypersensitivity reactions associated with platinum-containing antineoplastic agents for thoracic malignancies. Anticancer. Res. 31, 4525-4528.

Wang, H. B., Shen, K., Yang, J. X., Huang, H. F., Li, Y., Wu, M., et al. (2009). Clinical analysis of thirteen cases of hypersensitivity reactions to carboplatin. Zhonghua. Fu. Chan. Ke. Za. Zhi. 44, 837-840.

Zweizig, S., Roman, L. D., and Muderspach, L. I. (1994). Death from anaphylaxis to cisplatin: a case report. Gynecol. Oncol. 53, 121-122. doi: 10.1006/gyno.1994.1098

Conflict of Interest Statement: The authors declare that the research was conducted in the absence of any commercial or financial relationships that could be construed as a potential conflict of interest.

Copyright (c) 2017 Tai, Tai, Hsu, Lee, Chen, Chiang, Chen, Chen and Cheng. This is an open-access article distributed under the terms of the Creative Commons Attribution License (CC BY). The use, distribution or reproduction in other forums is permitted, provided the original author(s) or licensor are credited and that the original publication in this journal is cited, in accordance with accepted academic practice. No use, distribution or reproduction is permitted which does not comply with these terms. 